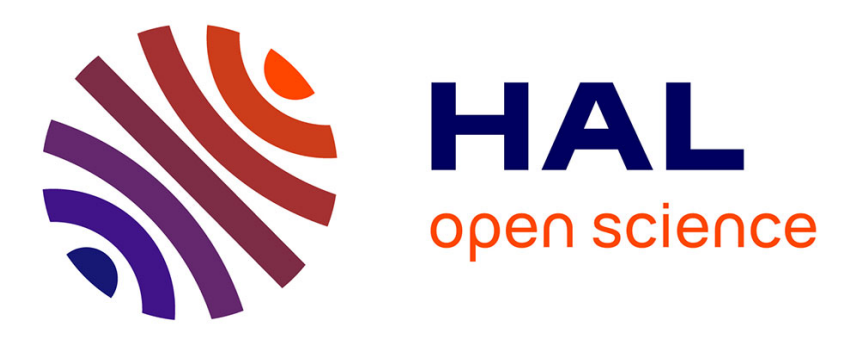

\title{
Saturation based nonlinear depth and yaw control of underwater vehicles with stability analysis and real-time experiments
}

Eduardo Campos Mercado, Ahmed Chemori, Vincent Creuze, Jorge Antonio Torres Muñoz, Rogelio Lozano

\section{To cite this version:}

Eduardo Campos Mercado, Ahmed Chemori, Vincent Creuze, Jorge Antonio Torres Muñoz, Rogelio Lozano. Saturation based nonlinear depth and yaw control of underwater vehicles with stability analysis and real-time experiments. Mechatronics, 2017, 45, pp.49-59. 10.1016/j.mechatronics.2017.05.004 . lirmm-01567465

\section{HAL Id: lirmm-01567465 https://hal-lirmm.ccsd.cnrs.fr/lirmm-01567465}

Submitted on 10 Sep 2019

HAL is a multi-disciplinary open access archive for the deposit and dissemination of scientific research documents, whether they are published or not. The documents may come from teaching and research institutions in France or abroad, or from public or private research centers.
L'archive ouverte pluridisciplinaire HAL, est destinée au dépôt et à la diffusion de documents scientifiques de niveau recherche, publiés ou non, émanant des établissements d'enseignement et de recherche français ou étrangers, des laboratoires publics ou privés. 


\title{
Saturation Based Nonlinear Depth and Yaw Control of Underwater Vehicles with Stability Analysis and Real-time Experiments
}

\author{
E. Campos ${ }^{2,3 *}$, A. Chemori ${ }^{3}$, V. Creuze ${ }^{3}$, J. Torres ${ }^{1,2}$, R. Lozano $^{2}$. \\ ${ }^{1}$ Automatic Control Department, CINVESTAV, México D.F., México \\ ${ }^{2}$ UMI-LAFMIA,CINVESTAV-CNRS, México, D.F., México \\ ${ }^{3}$ LIRMM, CNRS-Université Montpellier 2, Montpellier, France
}

\begin{abstract}
This paper deals with two nonlinear controllers based on saturation functions with varying parameters, for set-point regulation and trajectory tracking on an Underwater Vehicle. The proposed controllers combine the advantages of robust control and easy tuning in real applications. The stability of the closed-loop system with the proposed nonlinear controllers is proven by Lyapunov arguments. Experimental results for the trajectory tracking control in 2 degrees of freedom, these are the depth and yaw motion of an underwater vehicle, show the performance of the proposed control strategy.
\end{abstract}

Keywords: Underwater vehicle, Nonlinear PD and PD+ Controller, Saturation, Real-time experiments.

\section{INTRODUCTION}

Underwater vehicles are more and more used for various types of applications, such as inspection, exploration, oceanography, biology, to name a few. They can be classified in two classes: the Autonomous Underwater Vehicles (AUVs) and the Remotely Operated Vehicles (ROVs). One of the main challenges for these types of vehicles lies in the design of the control strategy, given the nonlinear dynamics and the difficulty to accurately identify their hydrodynamic parameters [2][3][4]. The controller is used either to fully control the vehicle (for AUVs), or to assist the pilot (for ROVs) by providing features such as auto-depth, auto-altitude (with respect to the seabed), or autoheading. Although many types of controllers have been studied during last decades, most of commercial underwater vehicles use PID controllers. For instance, PID control and acceleration feedback can be found in [5]; in [7] a PD controller considering the time-delay produced by the sensor has been proposed for an underwater vehicle. Nevertheless the drawback of these controllers is that they do not have a good performance when the parameters of the system change.

In practical applications, we can notice that a standard PID control design can be improved by bounding its signal. Consequently, several nonlinear PID controllers with bounded signal have been proposed in order to improve the performance of the closed-loop system. For instance, in [8] a nonlinear PD controller has been proposed for robot manipulators, where the constant proportional and derivative gains have been replaced with nonlinear functions. In [9] a nonlinear PID controller is

${ }^{*}$ Corresponding author. Tel.:+521 55857801 67; fax: (+52 55) 57473982. E-mail address: camposme1a@hotmail.com; ecampos@ctrl.cinvestav.mx.

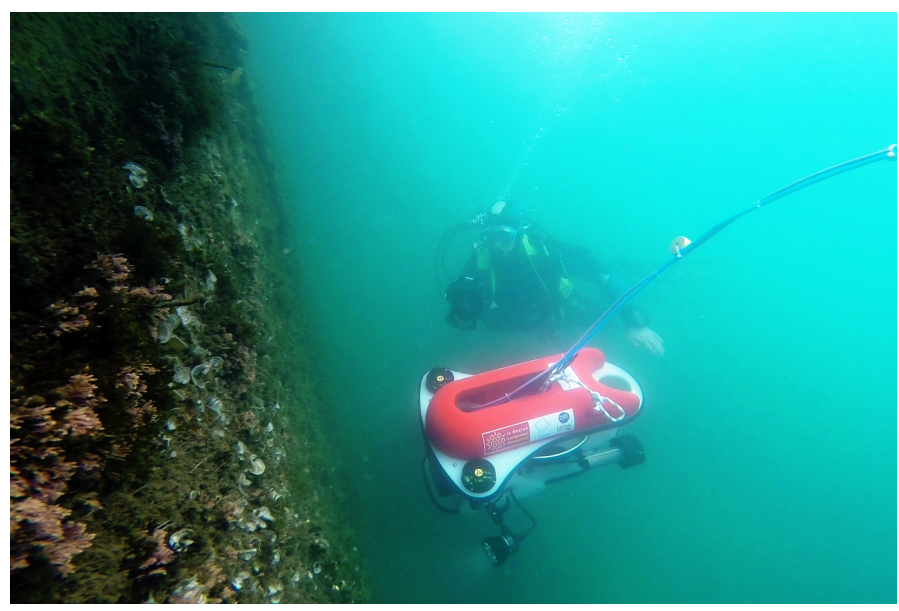

Figure 1: View of the L2ROV underwater vehicle. Its six thrusters allow precise control of its 6 degrees of freedom

proposed for a superconducting magnetic energy storage, where the idea was to improve the stability of the power system in a relatively wide operation range. In [10] a nonlinear PID controller was applied to a class of truck ABS (Anti-lock Brake System), where it has been shown that the nonlinear PID controller has better performance than the conventional PID controller.

In the literature there are some works about control strategies for AUVs, for example in the paper [11] the authors present a trajectory tracking control using a linear system to implement a sliding mode controller. In this case the unmodeled dynam- 
ics are consider as external perturbations. In [12] the simulation of a back-stepping controller for robust diving against pitch perturbations is given. The reference [13] describes a classical algorithm of sliding mode, where the vehicle has a input/output decentralized dynamics; the main problem of this technic is the chattering. The paper [15] presents a trajectory tracking control using Lagrange's operators, allowing propose a novel path-following controller for UUVs. Concerning robust controllers, one possibility is to try to reduce undesirable dynamic couplings, for instance dynamic pitch and yaw coupling suppression using a robust $H_{\infty}$ control technique has been considered in [16].

In the present paper, our aim is to reinforce the prominent place PD controllers have gained in a number of applications. In this vein, we propose a nonlinear PD and PD+ based on saturation function with variable parameters. Both controllers are proposed for set-point regulation as well as time varying trajectory tracking control of an Underwater Vehicle. To the best knowledge of the authors, this method has never been applied yet to control this type of vehicles. Moreover the proof of stability, based on Lyapunov arguments, is given and the control scheme is validated on a new underwater vehicle. Furthermore the experimental results presented herein have been extended to two degrees of freedom, namely depth and yaw.

The real-time experiments have been conducted using the tethered underwater vehicle L2ROV (Figure 1 and 2) entirely designed and built at LIRMM (University Montpellier 2). One of the main advantages of this vehicle is that we can use it either as an Autonomous Underwater Vehicle (AUV) or as a Remotely Operated Vehicle (ROV), depending on the task we want to carry out. The propulsion system consists of six thrusters used to control the 6-DOF, although roll and pitch are naturally stable. This paper is organized as follows: in section 2 we briefly describe the L2ROV prototype as well as its dynamic model. The control strategy is presented in section 3. The obtained experimental results for trajectory tracking control are presented and discussed in section 4. Finally, some concluding remarks and future works are given in section 5 .

\section{DESCRIPTION AND MODELING OF THE L2ROV VEHICLE}

This section describes the technical features of the L2ROV underwater vehicle and its dynamic model. Based on the design of the vehicle and in order to reduce further analysis, we assume that the vehicle is moving at low speeds, leading to a slightly simplified dynamics.

\subsection{Prototype description}

The L2ROV (Figure 1 and 2) is a tethered underwater vehicle, whose size is about $75 \mathrm{~cm}$ long, $55 \mathrm{~cm}$ width, and $45 \mathrm{~cm}$ height. The propulsion system of this underwater vehicle consists of six thrusters, as illustrated in Figure 2. According to the SNAME notation [17], the translational motions are referred to as surge, sway, and heave; while the rotational motions are roll, pitch, and yaw. The surge motion is generated by the sum of the forces created by $T_{4}$ and $T_{5}$, sway movement is actuated by $T_{6}$, and heave is produced by the sum of thrusts of $T_{1}$, $T_{2}$ and $T_{3}$. The roll movement is actuated through differential force of the thrusters $T_{2}$ and $T_{3}$; the pitch motion is obtained similarly using thrusters $T_{1}, T_{2}$ and $T_{3}$, and the yaw motion is generated by $T_{4}$ and $T_{5}$. The experimental platform consists

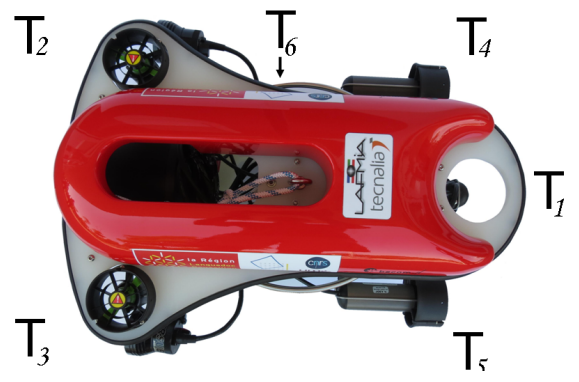

Figure 2: L2ROV: View of forces generated by the thrusters to perform the translational and rotational motions.

of a ROV driven by a laptop computer, with CPU Intel Core i7-3520M 2.9GHz, 8GB of RAM memory. The computer runs under Windows 7 operating system and the control software is developed with Visual C++ 2010. The computer receives the data from the ROV's sensors (pressure, attitude), computes the control laws and sends input signals to the actuators. These latter are controlled by MD03 Motor Drives. The main features of this vehicle are described in Table 1.

Table 1: The main features of the L2ROV vehicle

\begin{tabular}{ll}
\hline \hline Mass & $28 \mathrm{~kg}$ \\
Floatability & $9 \mathrm{~N}$ \\
Dimensions & $75 \mathrm{~cm}(\mathrm{l}) \times 55 \mathrm{~cm}(\mathrm{w}) \times 45 \mathrm{~cm}(\mathrm{~h})$ \\
Maximal depth & $100 \mathrm{~m}$ \\
Thrusters & 6 Seabotix BTD150 \\
& cont. bollard thrust = 2.2kgf each \\
& with Devantech MD03 drivers \\
Power & 48V - 600W \\
Light & 2 x 50W LED \\
Attitude sensor & Sparkfun Arduimu V3 \\
& Invensense MPU-6000 MEMS 3-axis gyro \\
& and accelerometer \\
& 3-axis I2C magnetometer HMC-5883L \\
& Atmega328 microprocessor \\
Camera & Pacific Corporation VPC-895A \\
& CCD1/3" PAL -25-fps \\
Depth sensor & Pressure Sensor Breakout-MS5803-14BA \\
Sampling period & 50ms \\
Surface computer & Dell Latitude E6230 - Intel Core i7 - 2.9GHz \\
& Windows 7 Professional 64 bits \\
Tether length & Microsoft Visual C++ 2010 \\
\hline
\end{tabular}




\subsection{Dynamic Modeling}

The dynamics of the vehicle, in the body-fixed-frame $\left(x_{b}, y_{b}, z_{b}\right)$ (more details see Figure 3), can be expressed in a compact matrix form as [18]:

$$
\begin{gathered}
M \dot{\nu}+C(\nu) \nu+D(\nu) \nu+g(\eta)=\tau+w_{e} \\
\dot{\eta}=J(\eta) \nu
\end{gathered}
$$

where $M \in \mathbb{R}^{6 \times 6}$ is the inertia matrix, $C(\nu) \in \mathbb{R}^{6 \times 6}$ defines the Coriolis-centripetal matrix. In our case we assume that the vehicle is moving at low speeds, then this Coriolis matrix can be neglected. $\boldsymbol{D}(\boldsymbol{\nu}) \in \mathbb{R}^{6 \times 6}$ represents the damping matrix, $\boldsymbol{g}(\boldsymbol{\eta}) \in \mathbb{R}^{6 \times 1}$ describes the vector of restoring forces and moments, $\boldsymbol{\tau}=\left(\boldsymbol{\tau}_{\mathbf{1}}, \boldsymbol{\tau}_{\mathbf{2}}\right)^{T}=\left(\left(\tau_{X}, \tau_{Y}, \tau_{Z}\right),\left(\tau_{K}, \tau_{M}, \tau_{N}\right)\right)^{T} \in \mathbb{R}^{6 \times 1}$ defines the vector of control inputs; $\boldsymbol{w}_{e} \in \mathbb{R}^{6 \times 1}$ defines the vector of disturbances; $\boldsymbol{\nu}=\left(\boldsymbol{\nu}_{1}, \boldsymbol{\nu}_{2}\right)^{T}=((u, v, w),(p, q, r))^{T} \in \mathbb{R}^{6 \times 1}$ represents the linear and angular velocity vector in the bodyfixed-frame; $\boldsymbol{\eta}=\left(\boldsymbol{\eta}_{1}, \boldsymbol{\eta}_{2}\right)^{T}=((x, y, z),(\phi, \theta, \psi))^{T} \in \mathbb{R}^{6 \times 1}$ is the position and attitude vector decomposed in the earth-fixedframe, and $\boldsymbol{J}(\boldsymbol{\eta}) \in \mathbb{R}^{6 \times 6}$ is the transformation matrix mapping from the body-fixed-frame to earth-fixed-frame (see Figure 3). For more details about the dynamic modeling, the reader can refer to [19],[20].

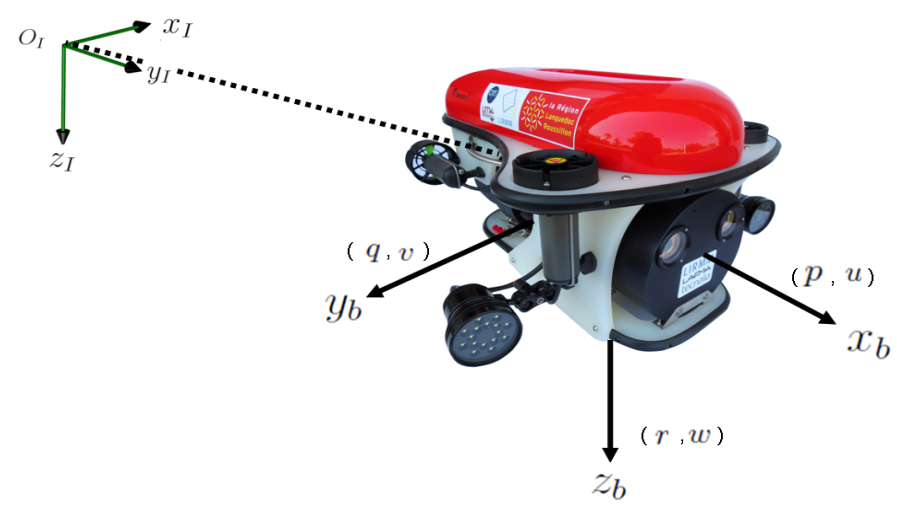

Figure 3: The L2ROV vehicle, with the body-fixed-frame $\left(O_{b}, x_{b}, y_{b}, z_{b}\right)$, and the earth-fixed-frame $\left(O_{I}, x_{I}, y_{I}, z_{I}\right)$.

\subsubsection{Inertia and Damping Matrices}

The inertia matrix $M$ is the sum of the rigid-body inertia $M_{R B}$ and the inertia of the added mass $M_{A}$, as follows:

$$
M=M_{R B}+M_{A}
$$

In our case, we assume that the vehicle is moving at slow speeds; hence, the $M$ matrix can be approximated by:

$$
\begin{aligned}
M=\operatorname{diag}\left\{m-X_{\dot{u}}, m-Y_{\dot{v}}, m-Z_{\dot{w}},\right. \\
\left.I_{x x}-K_{\dot{p}}, I_{y y}-M_{\dot{q}}, I_{z z}-N_{\dot{r}}\right\}
\end{aligned}
$$

where $m$ is the mass of the vehicle, $X_{\dot{u}}, Y_{\dot{v}}, Z_{\dot{w}}, K_{\dot{p}}, M_{\dot{q}}, N_{\dot{r}}$ represent hydrodynamic added mass, and $I_{x x}, I_{y y}, I_{z z}$ are the moments of inertia of the rigid-body. L2ROV inertia parameters (computed from the ROV's 3D model), then we have obtained the following values in $\mathrm{kg} \cdot \mathrm{m}^{2}$ :

$$
\boldsymbol{I}=\left[\begin{array}{ccc}
0.35 & -0.02 & -0.04 \\
-0.02 & 0.69 & -0.02 \\
-0.04 & -0.02 & 0.65
\end{array}\right]
$$

Concerning the hydrodynamic damping, we consider the damping model for low-speed underwater vehicles. Thus we have:

$$
D(\nu)=\operatorname{diag}\left\{X_{u}, Y_{v}, Z_{w}, K_{p}, M_{q}, N_{r}\right\}
$$

For the L2ROV prototype the damping parameters included in the damping matrix have been experimentally estimates by applying the following procedure. First, the buoyancy of the ROV is adjusted to exactly compensate for the weight, so that the floatability is neutral. Then, a known force is applied to the $\mathrm{ROV}$ along the $\mathrm{z}$ axis. This force is produced by the thrusters and is known thanks to a previous calibration. As the vehicle submerses, the value of $\mathrm{z}$ is recorded ( thanks to the depth sensor). Then, the speed along $\mathrm{z}$ is computed. After few seconds, the ROV reaches a steady state limit speed. The value of $Z_{w}$, the damping parameter along $\mathrm{z}$, is approximated by: $Z_{w} \simeq f_{z} / w_{\text {lim }}$, where $f_{z}$ is the force exerted by the thrusters along $\mathrm{z}$, and $w_{\text {lim }}$ is the linear speed of the ROV along $\mathrm{z}$. The estimated value of $Z_{w}$ is 80 N.s.m $m^{-1}$.

According to the symmetry of the vehicle, we consider that $Y_{v}$ is roughly equal to $Z_{w}$. The value of $X_{u}$ is computed by measuring the time needed by the ROV to run a known horizontal distance in a pool, with a known horizontal thrust. Then, the speed is computed and the damping parameter is estimated. The estimated value of $X_{u}$ is 30 N.s.m ${ }^{-1}$. Regarding the rotational damping parameter, we applied a known torque along $\mathrm{z}$ axis with the thrusters and we recorded the rate of turn measured by the gyrometer (along $\mathrm{z}$ axis) of the embedded IMU. Once the rate of turn reaches its steady state value $r_{l i m}$, the rotational damping parameter $N_{r}$ is approximated by: $N_{r} \simeq_{z} / r_{\text {lim }}$, where $\gamma_{z}$ is the applied torque.

The estimated value of $N_{r}$ is 2.9 N.m.s.rad ${ }^{-1}$. The symmetry of the L2ROV vehicle allows us to consider that $M_{q}$ is roughly equal to $N_{r}$. The value of $K_{p}$ (along x axis) has not be experimentally estimated as this would require to make the center of gravity coincide with the center of buoyancy. This is long and useless, since in our case the roll is naturally stable and is not controlled. According to the previous values and the geometry of the vehicle, we have considered that $K_{p} \simeq 1.4$ N.m.s.rad ${ }^{-1}$. Please note that we have assumed that the speed of the vehicle is sufficiently low to consider only the skin friction effects. Thus, we estimate only linear damping. Would the speed be higher, then quadratic damping would be taken into account and quadratic damping parameters should be computed by the same method, replacing each speed by its squared value. Given that the vehicle is moving slow and then, non diagonal terms 
of the damping matrix are neglected and only linear damping parameters have been estimated for this prototype, then

$$
\boldsymbol{D}(\boldsymbol{\nu})=\operatorname{diag}\{30,70,80,1.4,2.5,2.9\}
$$

in $\left(\frac{N . s}{m}\right)$ (first three) and in $\left(\frac{N . s}{r a d}\right)$ (last three).

\subsubsection{Restoring Forces and Moments}

The restoring forces and moments are generated by the weight $\boldsymbol{f}_{W}$ and the buoyancy force $\boldsymbol{f}_{\boldsymbol{B}}$, this latter, always acts in the opposite direction of vehicle weight, that is:

$$
\boldsymbol{f}_{B}=-\left[\begin{array}{c}
0 \\
0 \\
B
\end{array}\right] \quad \boldsymbol{f}_{W}=\left[\begin{array}{c}
0 \\
0 \\
W
\end{array}\right]
$$

where $B$ represents the magnitude of the buoyancy force, defined according to the Archimedes' principle; $W=m g$ is the vehicle's weight, with $g$ the gravitational acceleration. Notice that these forces are defined with respect to the earth-fixed-frame. Now, using the zyx-convention for navigation and control applications [5], the transformation matrix $J_{\mathbf{1}}\left(\eta_{\mathbf{2}}\right)=R_{z, \psi} R_{y, \theta} R_{x, \phi}$ is introduced in order to obtain the buoyancy force and weight with respect to the body-fixed-frame:

$$
F_{B}=J_{1}\left(\eta_{2}\right)^{-1} f_{B} \quad, \quad F_{W}=J_{1}\left(\eta_{2}\right)^{-1} f_{W}
$$

Then, the restoring forces acting on the vehicle are $\boldsymbol{f}_{g}=\boldsymbol{F}_{\boldsymbol{B}}+\boldsymbol{F}_{W}$, leading to:

$$
\boldsymbol{f}_{\boldsymbol{g}}=\left[\begin{array}{c}
(B-W) \sin (\theta) \\
(W-B) \cos (\theta) \sin (\phi) \\
(W-B) \cos (\theta) \cos (\phi)
\end{array}\right]
$$

On the other hand, the restoring moments depend on the positions of the center of gravity (CG) and the center of buoyancy $(\mathrm{CB})$, as we can notice in the following equation:

$$
m_{g}=r_{w} \times F_{W}+r_{b} \times F_{B}
$$

where $\boldsymbol{r}_{\boldsymbol{w}}=\left[x_{w}, y_{w}, z_{w}\right]^{T}$ and $\boldsymbol{r}_{\boldsymbol{b}}=\left[x_{b}, y_{b}, z_{b}\right]^{T}$ represent the positions of the center of gravity and the center of buoyancy, respectively. In our case the origin of the body-fixed-frame is chosen in the center of gravity, this implies that $\boldsymbol{r}_{\boldsymbol{w}}=[0,0,0]^{T}$, while the center of buoyancy is $\boldsymbol{r}_{\boldsymbol{b}}=\left[0,0,-z_{b}\right]^{T}$. For practical purposes, the buoyancy force is greater than the weight, i.e. $B-$ $W=f_{b}>0$. Then, from equations (10) and (11), we obtain the vector of restoring forces and moments as follows:

$$
\boldsymbol{g}(\boldsymbol{\eta})=\left[\begin{array}{c}
\boldsymbol{f}_{\boldsymbol{g}} \\
\boldsymbol{m}_{\boldsymbol{g}}
\end{array}\right]=\left[\begin{array}{c}
f_{b} \sin (\theta) \\
-f_{b} \cos (\theta) \sin (\phi) \\
-f_{b} \cos (\theta) \cos (\phi) \\
-z_{b} B \cos (\theta) \sin (\phi) \\
-z_{b} B \sin (\theta) \\
0
\end{array}\right]
$$

\subsubsection{Control Inputs: Forces and torques generated by the thrusters}

The forces generated by the thrusters $T_{1}$ to $T_{6}$ are denoted $\boldsymbol{f}_{\mathbf{1}}$ to $\boldsymbol{f}_{\mathbf{6}}$, and are defined by: $\boldsymbol{f}_{\mathbf{1}}=\left[0,0, f_{1}\right]^{T}, \boldsymbol{f}_{\mathbf{2}}=\left[0,0, f_{2}\right]^{T}$, $\boldsymbol{f}_{\mathbf{3}}=\left[0,0, f_{3}\right]^{T}, \boldsymbol{f}_{4}=\left[f_{4}, 0,0\right]^{T}, \boldsymbol{f}_{\mathbf{5}}=\left[f_{5}, 0,0\right]^{T}, \boldsymbol{f}_{6}=\left[0, f_{6}, 0\right]^{T}$, as illustrated in Figure 2. Then, the translation motions are produced by:

$$
\boldsymbol{\tau}_{\mathbf{1}}=\left[\begin{array}{c}
\tau_{X} \\
\tau_{Y} \\
\tau_{Z}
\end{array}\right]=\left[\begin{array}{c}
f_{4}+f_{5} \\
f_{6} \\
f_{1}+f_{2}+f_{3}
\end{array}\right]
$$

and the torques generated by the above forces, are defined as follows:

$$
\tau_{\mathbf{2}}=\sum_{i=1}^{6} \boldsymbol{l}_{\boldsymbol{i}} \times \boldsymbol{f}_{\boldsymbol{i}}
$$

where $\boldsymbol{l}_{\boldsymbol{i}}=\left(l_{i x}, l_{i y}, l_{i z}\right)$ is the position vector describing where the $\boldsymbol{f}_{\boldsymbol{i}}$ (for $i=1, . ., 6$.) forces apply, with respect to the bodyfixed reference frame. The torques generated by the thrusters are then described by:

$$
\boldsymbol{\tau}_{\mathbf{2}}=\left[\begin{array}{c}
\tau_{K} \\
\tau_{M} \\
\tau_{N}
\end{array}\right]=\left[\begin{array}{c}
l_{2 y} f_{2}+l_{3 y} f_{3} \\
l_{2 x} f_{2}+l_{3 x} f_{3}+l_{1 x} f_{1} \\
l_{4 y} f_{4}+l_{5 y} f_{5}
\end{array}\right]
$$

Finally, the vector of control inputs is expressed as follows:

$$
\boldsymbol{\tau}=\left[\begin{array}{c}
f_{4}+f_{5} \\
f_{6} \\
f_{1}+f_{2}+f_{3} \\
l_{2 y} f_{2}+l_{3 y} f_{3} \\
l_{2 x} f_{2}+l_{3 x} f_{3}+l_{1 x} f_{1} \\
l_{4 y} f_{4}+l_{5 y} f_{5}
\end{array}\right]
$$

\section{PROPOSED CONTROL STRATEGY}

In this section, nonlinear PD and PD+ controllers based on saturation functions with variable parameters are introduced. Both of them are proposed for set point regulation as well as for trajectory tracking control. The stability analysis of the resulting closed-loop system for both cases is detailed.

\subsection{Nonlinear PD Controller With Gravity and Buoyancy Com- pensation}

Considering the dynamics given by equations (1) and (2), the PD control law with static feedback gains and gravity/buoyancy compensation is given by:

$$
\tau=g(\eta)-J^{T}(\eta) \tau_{P D}
$$

with

$$
\tau_{P D}=K_{p} e(t)+K_{d} \frac{d e(t)}{d t}
$$

where $\boldsymbol{K}_{\boldsymbol{p}}, \boldsymbol{K}_{\boldsymbol{d}} \in \mathbb{R}^{6 \times 6}$ are diagonal, positive definite matrices, and $\boldsymbol{e}(\boldsymbol{t})=\boldsymbol{\eta}-\boldsymbol{\eta}_{\boldsymbol{d}}$ represents the error.

In order to improve the performance of the closed-loop system, we propose to introduce (in each term of equation (18)) a 
saturation function $\sigma_{\bar{b}}(h)$ illustrated in Figure 4 and defined by:

$$
\sigma_{\bar{b}}(h)=\left\{\begin{array}{cll}
\bar{b} & \text { if } & h>\bar{b} \\
h & \text { if } & |h| \leq \bar{b} \\
-\bar{b} & \text { if } & h<-\bar{b}
\end{array}\right.
$$

where $\bar{b}$ is a positive constant, and $h$ represents a linear function. In our case, the terms to which this saturation will be applied are the error and its time derivative.

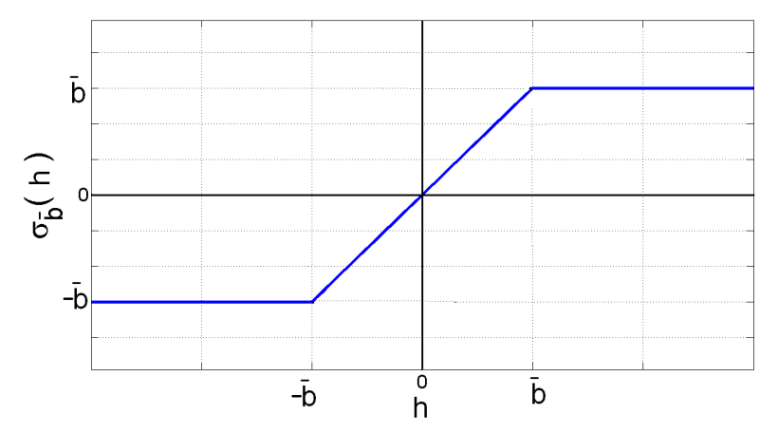

Figure 4: Saturation function with fixed parameters.

Then, if we introduce the above saturation function into in the control law (18), we obtain the following nonlinear PD (NLPD) controller:

$$
\tau_{N L P D}=\sigma_{\bar{b}_{p}}\left[K_{p} e(t)\right]+\sigma_{\bar{b}_{d}}\left[K_{d} \frac{d e(t)}{d t}\right]
$$

where

$$
\begin{gathered}
\boldsymbol{\sigma}_{\overline{\boldsymbol{b}}_{p}}\left[\boldsymbol{K}_{\boldsymbol{p}} \boldsymbol{e}(\boldsymbol{t})\right]=\left[\begin{array}{cccc}
u_{p 1} & 0 & \ldots & 0 \\
0 & u_{p 2} & \ldots & 0 \\
\vdots & \vdots & \ddots & \vdots \\
0 & 0 & \ldots & u_{p n}
\end{array}\right] \\
\boldsymbol{\sigma}_{\overline{\boldsymbol{b}}_{\boldsymbol{d}}}\left[\boldsymbol{K}_{\boldsymbol{d}} \frac{\boldsymbol{d e}(\boldsymbol{t})}{\boldsymbol{d} \boldsymbol{t}}\right]=\left[\begin{array}{cccc}
u_{d 1} & 0 & \ldots & 0 \\
0 & u_{d 2} & \ldots & 0 \\
\vdots & \vdots & \ddots & \vdots \\
0 & 0 & \ldots & u_{d n}
\end{array}\right]
\end{gathered}
$$

with $u_{p j}=\sigma_{\bar{b}_{p j}}\left[k_{p j} e_{j}(t)\right] ; u_{d j}=\sigma_{\bar{b}_{d j}}\left[k_{d j} \frac{d e_{j}(t)}{d t}\right]$; where $k_{p j}, k_{d j}$ are positive constants, for all $j=1$...n.

Without loss of generality, let us consider now the scalar case, namely:

$$
\tau_{N L P D 1}=\sigma_{\bar{b}_{p 1}}\left[k_{p 1} e_{1}(t)\right]+\sigma_{\bar{b}_{d 1}}\left[k_{d 1} \frac{d e_{1}(t)}{d t}\right]
$$

The above equation can be rewritten in a compact form as follows:

$$
\tau_{N L P D 1}=\sum_{i=1}^{2} u_{i}
$$

where $u_{i}=\sigma_{\bar{b}_{i}}\left(k_{i} h_{i}\right)$ represents the saturation function, with $b_{1}=\bar{b}_{p 1}, b_{2}=\bar{b}_{d 1}, k_{1}=k_{p 1}, k_{2}=k_{d 1} ; h_{1}$ is the error and $h_{2}$ its first derivative. Then, from equation (19) $u_{i}$ can be rewritten as:

$$
u_{i}=\left\{\begin{array}{cll}
\bar{b}_{i} & \text { if } & k_{i} h_{i}>\bar{b}_{i} \\
k_{i} h_{i} & \text { if } & \left|k_{i} h_{i}\right| \leq \bar{b}_{i} \\
-\bar{b}_{i} & \text { if } & k_{i} h_{i}<-\bar{b}_{i}
\end{array}\right.
$$

In the above equation, we can notice that the linear function $k_{i} h_{i}$ is saturated by $\left|h_{i}\right|=\bar{b}_{i} / k_{i}$. At that time, we define:

$$
d_{i}:=\bar{b}_{i} / k_{i}
$$

Then, we can rewrite equation (25) as follows:

$$
u_{i}=\left\{\begin{array}{cll}
\operatorname{sign}\left(h_{i}\right) \bar{b}_{i} & \text { if } & \left|h_{i}\right|>d_{i} \\
\bar{b}_{i} d_{i}^{-1} h_{i} & \text { if } & \left|h_{i}\right| \leq d_{i}
\end{array}\right.
$$

where the tuning parameters of the controller are $b_{i}$ and $d_{i}, \forall$ $i=1,2$. Moreover, considering that we have:

$$
\operatorname{sign}\left(h_{i}\right) \bar{b}_{i}=h_{i} \operatorname{sign}\left(h_{i}\right) \bar{b}_{i} h_{i}^{-1}
$$

which can be simplified as:

$$
\operatorname{sign}\left(h_{i}\right) \bar{b}_{i}=\left|h_{i}\right| \bar{b}_{i} h_{i}^{-1}
$$

and considering that $\left|h_{i}\right| h_{i}^{-1}=\left|h_{i}\right|^{-1} h_{i}$, equation (27) can be rewritten as follows:

$$
u_{i}=\left\{\begin{array}{ccc}
\bar{b}_{i}\left|h_{i}\right|^{-1} h_{i} & \text { if } & \left|h_{i}\right|>d_{i} \\
\bar{b}_{i} d_{i}^{-1} h_{i} & \text { if } & \left|h_{i}\right| \leq d_{i}
\end{array}\right.
$$

Consequently, the control law (23) can be rewritten as:

$$
\tau_{N L P D 1}=u_{1}+u_{2}=k_{p 1}(\cdot) e_{1}(t)+k_{d 1}(\cdot) \dot{e}_{1}(t)
$$

with:

$$
\begin{aligned}
& k_{p 1}(\cdot)=\left\{\begin{array}{cll}
\bar{b}_{p 1}\left|e_{1}(t)\right|^{-1} & \text { if } & \left|e_{1}(t)\right|>d_{p 1} \\
\bar{b}_{p 1} d_{p 1}^{-1} & \text { if } & \left|e_{1}(t)\right| \leq d_{p 1}
\end{array}\right. \\
& k_{d 1}(\cdot)=\left\{\begin{array}{cll}
\bar{b}_{d 1}\left|\dot{e}_{1}(t)\right|^{-1} & \text { if } & \left|\dot{e}_{1}(t)\right|>d_{d 1} \\
\bar{b}_{d 1} d_{d 1}^{-1} & \text { if } & \left|\dot{e}_{1}(t)\right| \leq d_{d 1}
\end{array}\right.
\end{aligned}
$$

The advantage of this formulation is that the forces and torques are limited by the parameters $\bar{b}_{p 1}$ and $\bar{b}_{d 1}$. Consequently, we are sure of the boundedness of the control input. However, some cases may require slightly larger forces and torques to correct the system errors, that is why we propose that the saturation value $\bar{b}_{i}$ in equation (30) should be changed as follows:

$$
\bar{b}_{i}=b_{i}\left|h_{i}\right|^{\mu_{i}} \quad \text { if } \quad\left|h_{i}\right|>d_{i}
$$

and

$$
\bar{b}_{i}=b_{i}\left|d_{i}\right|^{\mu_{i}} \quad \text { if } \quad\left|h_{i}\right| \leq d_{i}
$$


with $b_{i}$ a positive constant, and $\mu_{i} \in[0,1]$.

Now, introducing equations (34) and (35) into (30), we obtain:

$$
\begin{gathered}
u_{i}=\left\{\begin{array}{ccc}
b_{i}\left|h_{i}\right|^{\mu_{i}}\left|h_{i}\right|^{-1} h_{i} & \text { if } \quad\left|h_{i}\right|>d_{i} \\
b_{i}\left|d_{i}\right|^{\mu_{i}} d_{i}^{-1} h_{i} & \text { if } \quad\left|h_{i}\right| \leq d_{i}
\end{array}\right. \\
\forall i=1,2 \text { and } \mu_{i} \in[0,1] .
\end{gathered}
$$

The plots of the above function for different values of the parameter $\mu_{i}$ are shown in Figure 5.

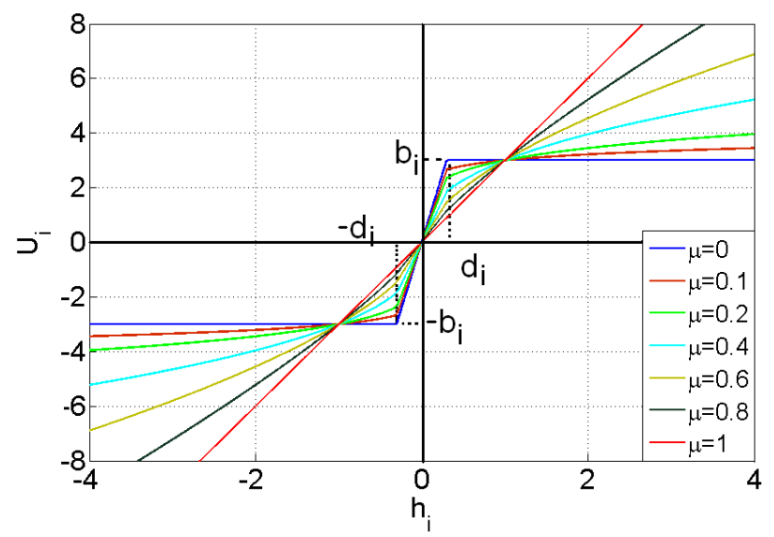

Figure 5: Saturation function with various values of parameter $\mu$.

Consequently, the nonlinear PD control law based on saturation function with variable parameters can be expressed as:

$$
\tau_{N L P D j}=k_{p j}(\cdot) e_{j}(t)+k_{d j}(\cdot) \dot{e}_{j}(t)
$$

with:

$$
\begin{gathered}
k_{p j}(\cdot)=\left\{\begin{array}{ccc}
b_{p j}\left|e_{j}(t)\right|^{\left(\mu_{p j}-1\right)} & \text { if } & \left|e_{j}(t)\right|>d_{p j} \\
b_{p j} d_{p j}^{\left(\mu_{p j}-1\right)} & \text { if } & \left|e_{j}(t)\right| \leq d_{p j}
\end{array}\right. \\
k_{d j}(\cdot)=\left\{\begin{array}{ccc}
b_{d j}\left|\dot{e}_{j}(t)\right|^{\left(\mu_{d j}-1\right)} & \text { if } & \left|\dot{e}_{j}(t)\right|>d_{d j} \\
b_{d j} d_{d j}^{\left(\mu_{d j}-1\right)} & \text { if } & \left|\dot{e}_{j}(t)\right| \leq d_{d j}
\end{array}\right. \\
\forall \mu_{p j}, \mu_{d j} \in[0,1]
\end{gathered}
$$

From Figure 5, it can be noticed that if $\mu_{p j}=\mu_{d j}=1$, the nonlinear PD controller given by (37) degenerates into the linear PD controller given by (18). Besides, if $\mu_{p j}=\mu_{d j}=0$, we obtain the case of a constant saturation. To summarize, we can conclude that the linear PD controller and the nonlinear PD controller with a simple saturation function, defined by equation (19), are particular cases of the proposed controller.
Theorem 1. For the case of set-point regulation, under the nonlinear PD control (NLPD) with gravity compensation

$$
\tau=g(\eta)-J^{T}(\eta)\left[K_{p}(\cdot) e+K_{d}(\cdot) \dot{e}\right]
$$

where the feedback gains $\boldsymbol{K}_{p}(\cdot)$ and $\boldsymbol{K}_{\boldsymbol{d}}(\cdot)$ have the following structure:

$$
\begin{aligned}
\boldsymbol{K}_{\boldsymbol{p}}(\cdot) & =\left[\begin{array}{cccc}
k_{p 1}(\cdot) & 0 & \ldots & 0 \\
0 & k_{p 2}(\cdot) & \ldots & 0 \\
\vdots & \vdots & \ddots & \vdots \\
0 & 0 & \ldots & k_{p n}(\cdot)
\end{array}\right]>0 \\
\boldsymbol{K}_{\boldsymbol{d}}(\cdot) & =\left[\begin{array}{cccc}
k_{d 1}(\cdot) & 0 & \ldots & 0 \\
0 & k_{d 2}(\cdot) & \ldots & 0 \\
\vdots & \vdots & \ddots & \vdots \\
0 & 0 & \ldots & k_{d n}(\cdot)
\end{array}\right]>0
\end{aligned}
$$

the system (1) is globally asymptotically stable if $k_{p j}(\cdot)$ and $k_{d j}(\cdot)$ are defined by (38) and (39) respectively.

PROOF. In the case of set-point regulation $\boldsymbol{\eta}_{\boldsymbol{d}}$ is constant, then $\dot{\eta}_{d}=0$ and $\dot{e}=\dot{\eta}$. As a consequence the control law given by equation (40) can be rewritten as:

$$
\boldsymbol{\tau}=\boldsymbol{g}(\boldsymbol{\eta})-\boldsymbol{J}^{T}(\boldsymbol{\eta})\left[\boldsymbol{K}_{\boldsymbol{p}}(\cdot) \boldsymbol{e}+\boldsymbol{K}_{\boldsymbol{d}}(\cdot) \dot{\boldsymbol{\eta}}\right]
$$

In what follows we will suppose that $\theta \neq \pm \pi / 2$, in order to avoid possible singularities of $\boldsymbol{J}(\boldsymbol{\eta})$ matrix, see [5]. Now assuming that $\boldsymbol{w}_{\boldsymbol{e}}=0$, the injection of the control law (43) into (1), leads to the following closed-loop system:

$$
M \dot{\nu}+C(\nu) \nu+D(\nu) \nu=-J^{T}(\eta)\left[K_{p}(\cdot) e+K_{d}(\cdot) \dot{\eta}\right]
$$

and if we consider the transformation (2), we obtain:

$$
M \dot{\nu}+C(\nu) \nu+D(\nu) \nu=-J^{T}(\eta)\left[K_{p}(\cdot) e+K_{d}(\cdot) J(\eta) \nu\right]
$$

Let us define $\boldsymbol{K}_{\boldsymbol{d d}}(\cdot)=\boldsymbol{J}^{\boldsymbol{T}}(\boldsymbol{\eta}) \boldsymbol{K}_{\boldsymbol{d}}(\cdot) \boldsymbol{J}(\boldsymbol{\eta})$, then the previous equation can be rewritten as:

$M \dot{\nu}+C(\nu) \nu+D(\nu) \nu=-J^{T}(\eta) K_{p}(\cdot) e-K_{d d}(\cdot) \nu$

The closed-loop system (46) can be represented as

$$
\frac{d}{d t}\left[\begin{array}{l}
e \\
\nu
\end{array}\right]=
$$




$$
\left[\begin{array}{c}
J(\eta) \nu \\
M^{-1}\left[-J^{T}(\eta) K_{p}(\cdot) e-K_{d d}(\cdot) \nu-C(\nu) \nu-D(\nu) \nu\right]
\end{array}\right]
$$

Notice that the origin of the state space model is a unique equilibrium point. Now, in order to proof the globally asymptotic stability of the closed-loop system we propose the following Lyapunov function candidate:

$$
V(e, \nu)=\frac{1}{2} \nu^{T} M \nu+\int_{0}^{e} \xi^{T} K_{p}(\xi) d \xi
$$

where

$$
\begin{array}{r}
\int_{\mathbf{o}}^{e} \boldsymbol{\xi}^{\boldsymbol{T}} \boldsymbol{K}_{\boldsymbol{p}}(\boldsymbol{\xi}) \boldsymbol{d} \boldsymbol{\xi}=\int_{0}^{e_{1}} \xi_{1} k_{p 1}\left(\xi_{1}\right) d \xi_{1}+\int_{0}^{e_{2}} \xi_{2} k_{p 2}\left(\xi_{2}\right) d \xi_{2}+ \\
\int_{0}^{e_{3}} \xi_{3} k_{p 3}\left(\xi_{3}\right) d \xi_{3}+\ldots+\int_{0}^{e_{n}} \xi_{n} k_{p n}\left(\xi_{n}\right) d \xi_{n} .
\end{array}
$$

Now, considering that the inequality

$$
e_{j} k_{p j}(\cdot) \geq \alpha_{j}\left(\left|e_{j}\right|\right)
$$

is satisfied with the class $\boldsymbol{K}$ functions

$$
\alpha_{j}\left(\left|e_{j}\right|\right)=\left\{\begin{array}{lll}
\frac{b_{j}\left|e_{j}\right|^{\mu_{p j}} e_{j}}{a+\left|e_{j}\right|} & \text { if } & \left|e_{j}\right|>d_{j} \\
\frac{b_{j} d_{j}^{\mu_{p j}} e_{j}}{a+d_{j}} & \text { if } & \left|e_{j}\right| \leq d_{j}
\end{array}\right.
$$

with $b_{p j}>b_{j}, a>0$ and $d_{p j}<d_{j}$. Then, according to Lemma 2 from [8] one deduces the following:

$$
\int_{0}^{e} \xi^{T} K_{p}(\xi) d \xi>0 \quad \forall e \neq 0 \in R^{n}
$$

and

$$
\int_{0}^{e} \xi^{T} K_{p}(\xi) d \xi \rightarrow \infty \quad \text { as } \quad\|e\| \rightarrow \infty
$$

Therefore, the Lyapunov function candidate $V(\boldsymbol{e}, \boldsymbol{\nu})$ is a globally positive definite and radially unbounded.

The time derivative of the Lyapunov function candidate is:

$$
\dot{V}(e, \nu)=\nu^{T} M \dot{\nu}+e^{T} K_{p}(e) J(\eta) \nu
$$

by substituting the closed-loop equation (46) into (53) one obtains:

$$
\begin{gathered}
\dot{V}(e, \nu)=-\nu^{T} J^{T}(\eta) K_{p}(e) e-\nu^{T} K_{d d}(\eta, \dot{e}) \nu- \\
\nu^{T} C(\nu) \nu-\nu^{T} D(\nu) \nu+e^{T} K_{p}(e) J(\eta) \nu
\end{gathered}
$$

since $\boldsymbol{K}_{p}(e)=\boldsymbol{K}_{p}^{\boldsymbol{T}}(\boldsymbol{e})$ and $\boldsymbol{C}(\boldsymbol{\nu})=-\boldsymbol{C}(\boldsymbol{\nu})^{\boldsymbol{T}}$, equation (54) becomes:

$$
\dot{V}(e, \nu)=-\nu^{T}\left[K_{d d}(\eta, \dot{e})+D(\nu)\right] \nu
$$

Recall that $\boldsymbol{K}_{\boldsymbol{d}}=\boldsymbol{K}_{\boldsymbol{d}}^{\boldsymbol{T}}>0$, therefore $\boldsymbol{K}_{\boldsymbol{d} \boldsymbol{d}}=\boldsymbol{K}_{\boldsymbol{d} \boldsymbol{d}}^{\boldsymbol{T}}>0$, and assuming that $\boldsymbol{D}(\boldsymbol{\nu})>0$, then one can conclude that $\dot{V}(\boldsymbol{e}, \boldsymbol{\nu})$ is a globally negative semidefinite. Therefore the stability of the equilibrium point is guaranteed. In order to prove the asymptotic stability, the Krasovskii-LaSalle's theorem can be used, let

$$
\boldsymbol{\Omega}=\left\{\left[\begin{array}{c}
\boldsymbol{e} \\
\boldsymbol{\nu}
\end{array}\right]: \dot{V}(\boldsymbol{e}, \boldsymbol{\nu})=0\right\}=\left\{\left[\begin{array}{l}
\boldsymbol{e} \\
\boldsymbol{\nu}
\end{array}\right]=\left[\begin{array}{l}
\boldsymbol{e} \\
0
\end{array}\right] \in \mathbb{R}^{2 n}\right\}
$$

introducing $\boldsymbol{\nu}=0$ and $\dot{\nu}=0$ into equation (46) leads to the unique invariant point $e=0$. Therefore, we conclude that equilibrium point is globally asymptotically stable.

\subsection{Nonlinear PD+ Controller}

For the case of trajectory tracking problem, we propose to use a nonlinear PD+ controller with the same feedback gains as the previous controller.

Based on equation (2), the following kinematic transformations can be obtained (see [5] for more details):

$$
\ddot{\boldsymbol{\eta}}=\boldsymbol{J}(\boldsymbol{\eta}) \dot{\boldsymbol{\nu}}+\dot{\boldsymbol{J}}(\boldsymbol{\eta}) \boldsymbol{\nu} \quad \Longrightarrow \dot{\boldsymbol{\nu}}=\boldsymbol{J}^{-\mathbf{1}}(\boldsymbol{\eta})\left[\ddot{\boldsymbol{\eta}}-\dot{\boldsymbol{J}}(\boldsymbol{\eta}) \boldsymbol{J}^{-\mathbf{1}}(\boldsymbol{\eta}) \dot{\boldsymbol{\eta}}\right]
$$

Applying the previous transformations to the dynamic model (1), one obtains:

$$
\begin{aligned}
M_{\boldsymbol{\eta}}(\boldsymbol{\eta}) & =\boldsymbol{J}^{-\boldsymbol{T}}(\boldsymbol{\eta}) \boldsymbol{M J ^ { - 1 }}(\boldsymbol{\eta}) \\
\boldsymbol{C}_{\boldsymbol{\eta}}(\boldsymbol{\nu}, \boldsymbol{\eta}) & =\boldsymbol{J}^{-\boldsymbol{T}}(\boldsymbol{\eta})\left[\boldsymbol{C}(\boldsymbol{\nu})-\boldsymbol{M} \boldsymbol{J}^{-\mathbf{1}}(\boldsymbol{\eta}) \dot{\boldsymbol{J}}(\boldsymbol{\eta})\right] \boldsymbol{J}^{-\mathbf{1}}(\boldsymbol{\eta}) \\
\boldsymbol{D}_{\boldsymbol{\eta}}(\boldsymbol{\nu}, \boldsymbol{\eta}) & =\boldsymbol{J}^{-\boldsymbol{T}}(\boldsymbol{\eta}) \boldsymbol{D}(\boldsymbol{\nu}) \boldsymbol{J}^{-\mathbf{1}}(\boldsymbol{\eta}) \\
\boldsymbol{g}_{\boldsymbol{\eta}}(\boldsymbol{\eta}) & =\boldsymbol{J}^{-\boldsymbol{T}}(\boldsymbol{\eta}) \boldsymbol{g}(\boldsymbol{\eta}) \\
\boldsymbol{\tau}_{\boldsymbol{\eta}}(\boldsymbol{\eta}) & =\boldsymbol{J}^{-\boldsymbol{T}}(\boldsymbol{\eta}) \boldsymbol{\tau}
\end{aligned}
$$

Consequently, the dynamic model (1) expressed in the earthfixed-frame becomes:

$$
M_{\eta}(\eta) \ddot{\eta}+C_{\eta}(\nu, \eta) \dot{\eta}+D_{\eta}(\nu, \eta) \dot{\eta}+g_{\eta}(\eta)=J^{-T}(\eta) \tau
$$

Theorem 2. For the case of the trajectory tracking control, the nonlinear $P D+$ controller $(N L P D+)$ :

$$
\begin{gathered}
\tau=-J^{T}(\eta)\left[M_{\eta}(\eta) \ddot{\eta}_{d}+C_{\eta}(\nu, \eta) \eta_{d}+D_{\eta}(\nu, \eta) \dot{\eta}_{d}\right. \\
\left.+g_{\eta}(\eta)+K_{p}(\cdot) e+K_{d}(\cdot) \dot{e}\right]
\end{gathered}
$$

where the matrices $\boldsymbol{K}_{\boldsymbol{p}}(\cdot)$ and $\boldsymbol{K}_{\boldsymbol{d}}(\cdot)$ have the following structure:

$$
\boldsymbol{K}_{\boldsymbol{p}}(\cdot)=\left[\begin{array}{cccc}
k_{p 1}(\cdot) & 0 & \ldots & 0 \\
0 & k_{p 2}(\cdot) & \ldots & 0 \\
\vdots & \vdots & \ddots & \vdots \\
0 & 0 & \ldots & k_{p n}(\cdot)
\end{array}\right]>0
$$




$$
\boldsymbol{K}_{\boldsymbol{d}}(\cdot)=\left[\begin{array}{cccc}
k_{d 1}(\cdot) & 0 & \ldots & 0 \\
0 & k_{d 2}(\cdot) & \ldots & 0 \\
\vdots & \vdots & \ddots & \vdots \\
0 & 0 & \ldots & k_{d n}(\cdot)
\end{array}\right]>0
$$

globally asymptotically stabilizes the system (1) if $k_{p j}(\cdot)$ and $k_{d j}(\cdot)$ are defined by (38) and (39) respectively.

Proof. Injecting the control law (58) in equation (57), leads to the following closed-loop system:

$$
M_{\eta}(\eta) \ddot{e}=-C_{\eta}(\nu, \eta) \dot{e}-D_{\eta}(\nu, \eta) \dot{e}-K_{p}(\cdot) e-K_{d}(\cdot) \dot{e}
$$

which can be rewritten as:

$$
\begin{gathered}
\frac{d}{d t}\left[\begin{array}{c}
e \\
\dot{e}
\end{array}\right]= \\
\dot{e} \\
{\left[\begin{array}{c}
M_{\eta}(\boldsymbol{\eta})^{-1}\left[\left[C_{\eta}(\nu, \eta)+D_{\eta}(\nu, \eta)+K_{d}(\cdot)\right] \dot{e}+K_{p}(\cdot) e\right]
\end{array}\right]}
\end{gathered}
$$

where it can be noticed that the resulting system is autonomous and the origin is its unique equilibrium point.

The stability analysis can be conducted in the same way as for the previous controller, then considering the following Lyapunov function candidate:

$$
V(e, \dot{e})=\frac{1}{2} \dot{e}^{T} M_{\eta}(\eta) \dot{e}+\int_{0}^{e} \xi^{T} K_{p}(\xi) d \xi
$$

and according to arguments used in proof of the previous section, we conclude that $V(e, \dot{e})$ is also globally positive definite and radially unbounded.

The time derivative of this Lyapunov function candidate gives:

$$
\dot{V}(e, \dot{e})=\dot{e}^{T} M_{\eta}(\eta) \ddot{e}+\frac{1}{2} \dot{e}^{T} \dot{M}_{\eta}(\eta) \dot{e}+e^{T} K_{p}(\cdot) \dot{e}
$$

Now, injecting equation (61) in (64) and assuming that $\dot{M}_{\eta}=0$, and $\boldsymbol{C}_{\boldsymbol{\eta}}(\boldsymbol{\nu}, \boldsymbol{\eta})$ is skew symmetric, then:

$$
\dot{V}(e, \dot{e})=-\dot{e}^{T}\left[D_{\eta}(\nu, \eta)+K_{d}(\cdot)\right] \dot{e}
$$

Assuming that $\boldsymbol{D}_{\boldsymbol{\eta}}(\boldsymbol{\nu}, \boldsymbol{\eta})>0$ and remembering that $\boldsymbol{K}_{\boldsymbol{d}}(\cdot)>0$ and symmetric matrix, we deduce that $\dot{V}(e, \dot{e})$ is globally negative semidefinite, and therefore we can conclude stability of the equilibrium point. In order to prove asymptotic stability we apply the Krasovskii-LaSalle's theorem. Consider the set $\boldsymbol{\Omega}$ defined as:

$$
\boldsymbol{\Omega}=\left\{\left[\begin{array}{c}
\boldsymbol{e} \\
\dot{e}
\end{array}\right]: \dot{V}(\boldsymbol{e}, \dot{e})=0\right\}=\left\{\left[\begin{array}{c}
\boldsymbol{e} \\
\dot{e}
\end{array}\right]=\left[\begin{array}{c}
\boldsymbol{e} \\
0
\end{array}\right] \in \mathbb{R}^{2 n}\right\}
$$

Introducing $\dot{\boldsymbol{e}}=0$ and $\ddot{\boldsymbol{e}}=0$ into equation (61), we deduce that the unique invariant set is defined by $e=0$. As a consequence we conclude that equilibrium point is globally asymptotically stable. Notices that in case of $\boldsymbol{\eta}_{\boldsymbol{d}}$ constant, the nonlinear PD+ controller degenerates to the nonlinear PD controller presented in the previous section.

Now, before to implementing the proposed control strategies it is important to know the behavior of the thrusters of the robot, consequently a serie of experiments were conducted to obtain the relationship describing the force generated by thrusters in terms of the supply voltage, as ilustrated in Figure 6. In some works we can notice that the curve describing the behavior of the thrusters is aproximately square, for instance in [1], [6] and [14]. In our case the thrusters of the vehicle have a good behavior, which can help us in the experimental tests.

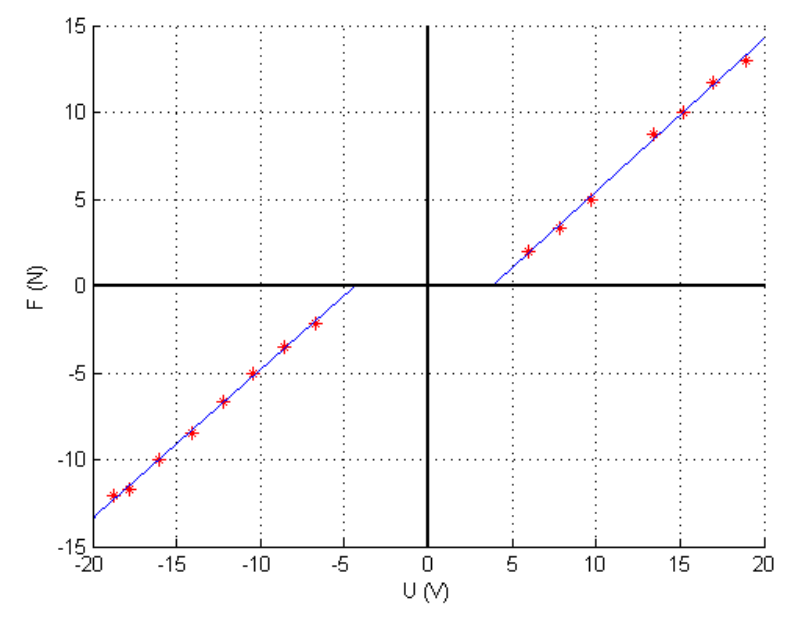

Figure 6: Evolution of the generated force $\mathrm{F}(\mathrm{N})$ by thrusters versus the supply voltage $\mathrm{U}(\mathrm{V})$

\section{REAL-TIME EXPERIMENTAL RESULTS}

Eventhough in this paper the stability analysis of the resulting closed-loop system with the proposed control strategy is addressed for the 6 degrees of freedom, the experimental results show only the behavior of two degrees of freedom, namely the yaw rotation and the depth traslation along the $\mathrm{z}$ axis. Since in a lots of applications we need the vehicle to be close to $\theta=0$ (pitch) and $\phi=0$ (roll), which is possible thanks to the design of the vehicle, therefore we have decided to control only the yaw motion. In the case of traslation motions we can control only the depth of the vehicle since it lacks a DVL or other sensor to estimate the positions $\mathrm{X}$ and $\mathrm{Y}$.

The experimental setup consists of a small swimming pool with a capacity of 3000 litters where the maximal depth is 1.2 $\mathrm{m}$. From an experimental point of view, the main control goal is the validation of the nonlinear PD and nonlinear PD+ controllers with variable saturation for depth and yaw tracking, in the same time. The idea is also to show the effectiveness of the 
proposed control solution against possible changes in the buoyancy and damping parameters that may occur during the experiments. The experimental results proposed hereafter have been conducted through the implementation of the proposed controllers on the of L2ROV underwater vehicle, you can watch the real-time experiments in: www.youtube.com/watch?v=SZZm4 He2-CA\&feature=youtu.be.

\subsection{Proposed experimental scenarios}

From theorem 3.2 we can notice that the nonlinear PD+ controller degenerate to a nonlinear PD controller when the desired trajectory is constant. Then, trajectory tracking control can be seen as an extension of set-point control. Moreover, from theorem 3.1, it can be concluded that the nonlinear PD+ controller becomes a PD+ controller when $\mu_{p j}=\mu_{d j}=1$. As a consequence, we consider implementing the nonlinear PD+ controller with the parameters' values summarized in Table 2, and the desired depth and yaw trajectories depicted in Figure 7 and Figure 8, respectively.

Table 2: Testing cases for the nonlinear PD+ controller

\begin{tabular}{lcc}
\hline \hline NLPD+ controller & Depth & Yaw \\
\hline Case 1 & $\mu_{p 3}=\mu_{d 3}=1$ & $\mu_{p 6}=\mu_{d 6}=1$ \\
Case 2 & $\mu_{p 3}, \mu_{d 3} \in[0,1]$ & $\mu_{p 6}, \mu_{d 6} \in[0,1]$ \\
\hline
\end{tabular}

Finally, in order to test the robustness of the proposed control schemes, the following scenarios are proposed for the previous cases:

\section{- SCENARIO 1 : Nominal Case}

The main goal of this scenario is to tune the controller gains in order to get the best trajectory tracking performance. The gains are kept unchanged for the scenario 2 .

- SCENARIO 2: Robustness towards uncertainties

The objective of this scenario is to test the robustness of the proposed controllers when vehicle's parameters (damping and buoyancy) are changed.

\subsection{Scenario 1: Nominal Case}

Given the characteristics of the control proposed in case 1 , the gains of the control have been tuned in two steps. The first one is based on the Integral of Squared Time multiplied by Squared Error (ISTSE) presented in [21]. In the second step the gains have been manually adjusted to get best results. The obtained parameters are summarized in Table 3.

The control parameters for the case 2 are given in Table 4, they are obtained by a heuristic method based on the following steps:

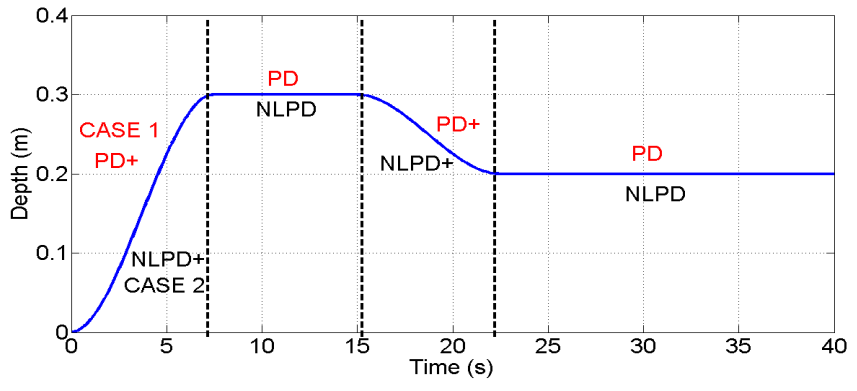

Figure 7: Evolution versus time of the desired trajectory for depth motions.

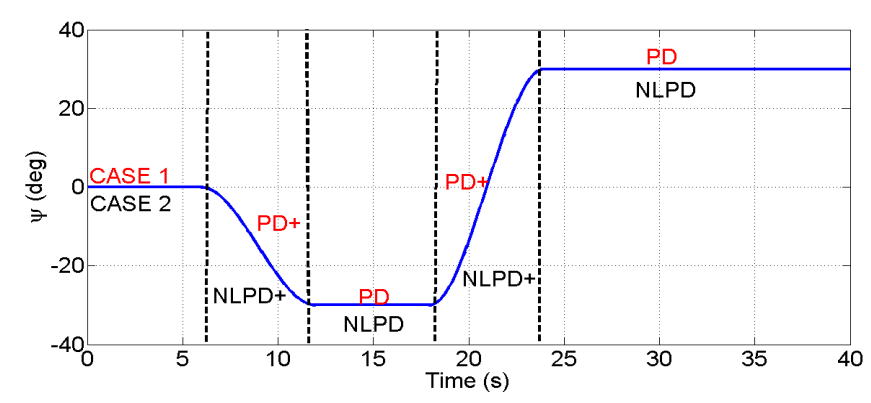

Figure 8: Evolution versus time of the desired trajectory for yaw motion.

- First $d_{p j}$ is chosen, taking into account that the interval $\left[-d_{p j}, d_{p j}\right]$ is the linear region of the proposed controller.

- Considering $b_{d j}=0$ and $\mu_{p j}=0 ; b_{p j}$ is increased until the closed-loop system oscillates.

- $d_{d j}$ is chosen bigger than $d_{p j}$, and $\mu_{d j}=0$.

- Then $b_{d j}$ is increased until the system oscillations decrease.

- Finally, $\mu_{p j}$ and $\mu_{d j}$ are adjusted to improve the system behavior, considering $\mu_{p j}<\mu_{d j}$.

Table 3: Parameters of the controller for case 1

\begin{tabular}{cccl}
\hline \hline Depth & $b_{p 3}=70$ & $d_{p 3}=\infty$ & $\mu_{p 3}=1$ \\
& $b_{d 3}=5$ & $d_{d 3}=\infty$ & $\mu_{d 3}=1$ \\
& & & \\
Yaw & $b_{p 6}=11$ & $d_{p 6}=\infty$ & $\mu_{p 6}=1$ \\
& $b_{d 6}=1.5$ & $d_{d 6}=\infty$ & $\mu_{d 6}=1$ \\
\hline
\end{tabular}

Figure 10-(a) shows the obtained results for trajectory tracking in depth, the corresponding tracking error, and the control input for the controller defined in Case 1. Figure 10-(b) shows the evolution of the tracking in yaw, the corresponding tracking error, and the control input produced by the thrusters.

Figure 11-(a) depicts the experimental results for trajectory tracking in depth, the corresponding tracking error, and the 
Table 4: Parameters of the controller for case 2

\begin{tabular}{lccc}
\hline \hline Depth & $b_{p 3}=20$ & $d_{p 3}=0.05$ & $\mu_{p 3}=0.1$ \\
& $b_{d 3}=13$ & $d_{d 3}=0.25$ & $\mu_{d 3}=0.2$ \\
& & & \\
Yaw & $b_{p 6}=4$ & $d_{p 6}=5.72$ & $\mu_{p 6}=0.09$ \\
& $b_{d 6}=5$ & $d_{d 6}=14.32$ & $\mu_{d 6}=0.2$ \\
\hline
\end{tabular}

control input for the controller defined in Case 2. Figure 11-(b) shows the evolution of the tracking in yaw, the corresponding tracking error and the yaw control input. Moreover, we can observe that the yaw motion converges to the desired trajectory in less than 1.5 seconds.

In order to evaluate the tracking performance of the proposed controllers, let us compute the Root Mean Square Error (RMSE) for $z$ and $\psi$. In addition, the integral of control inputs (the applied force and torque) are computed to estimated the energy consumption used in each case, that is:

$$
I N T=\int_{t_{1}}^{t_{2}}|\tau(t)| d t
$$

where $t_{1}=2$ seconds, since in this time for both cases the system's states are close to their desired values, and $t_{2}=30 \mathrm{sec}$ onds.

Table 5: Evaluation Criteria for scenario 1

\begin{tabular}{lcccc}
\hline \hline & $R M S E_{z}(m)$ & $I N T_{z}$ & $R M S E_{\psi}(\operatorname{deg})$ & $I N T_{\psi}$ \\
Case 1 & 0.0087 & 4657 & 0.04 & 507.2 \\
Case 2 & 0.0044 & 4913 & 0.03 & 647.6 \\
\hline
\end{tabular}

From the results of Table 5, we observe that the RMS $E_{z}$ and $R M S E_{\psi}$ of case 2 are smaller than in case 1 . It can be observed that steady-state errors $z$ and $\psi$ are approximately $0.8 \mathrm{~mm}$ and $0.04 \mathrm{deg}$ for the case 1 , while for the case 2 are approximately $0.4 \mathrm{~mm}$ and $0.03 \mathrm{deg}$ respectively. Moreover, notice that the quotients between $I N T_{z}$ and $I N T_{\psi}$ from case 1 and 2 are:

$$
\frac{4913}{4657}=1.0550 \quad \frac{647.6}{507.02}=1.27
$$

This means that energy consumption for trajectory tracking in depth, using the controller defined in Case 2, is 1.055 times the energy consumption using the controller defined in Case 1. While energy consumption for trajectory tracking in heading, using the controller defined in Case 2, is 1.27 times the energy consumption using the controller defined in Case 1.

\subsection{Scenario 2: Robustness Test}

The main goal of this scenario is to test the robustness of the proposed controllers towards uncertainties in the parameters of the model. During the real-time experiments, we have added two $200 \mathrm{~g}$ buoyant floats (as illustrated in Figure 9), increasing the buoyancy of $330 \%$, and a large $(45 \mathrm{~cm} \times 10 \mathrm{~cm})$ rigid plastic sheet (as illustrated in Figure 9), increasing the rotational damping along $\mathrm{z}$ axis of about $90 \%$.

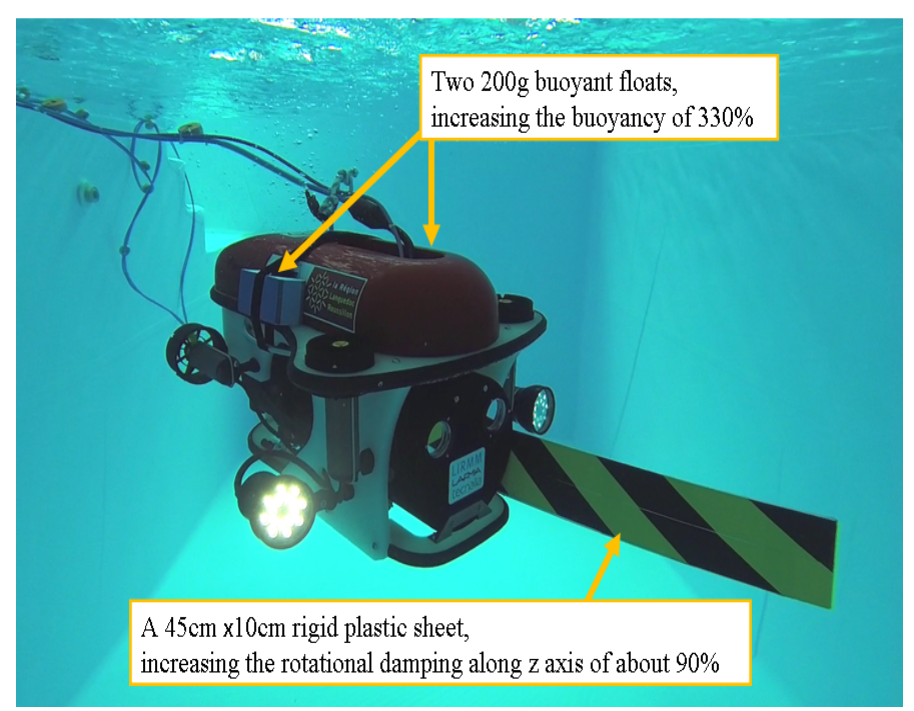

Figure 9: L2ROV with the added two buoyant floats and a rigid plastic sheet, which will increase the buoyancy force and damping along $\mathrm{z}$ axis.

The obtained experimental results for case 1 are shown in Figure 12. The tracking performance of the control system for depth is degraded. Indeed, depth control of the vehicle was not able to reach the desired trajectory. We can notice that steadystate error on $z$ and $\psi$ are approximately $11 \mathrm{~cm}$ and $0.01 \mathrm{deg}$, respectively. For the yaw motion, the vehicle converges to the desired trajectory in less than 1 second (as noticed in Figure 12-(a) ). The force $\tau_{z}$ and torque $\tau_{\psi}$ generated by the thrusters are displayed at the bottom curves of Figure 12.

Figure 13 shows that the performance of the system is less affected in case 2 . Indeed, it can be observed that the steadystate errors on $z$ and $\psi$ are approximately $6 \mathrm{~cm}$ and $0.006 \mathrm{deg}$, respectively. The yaw motion is the less affected, since the vehicle converge to the desired trajectory in less than 1 second. The generated control input (force $\tau_{z}$ and torque $\tau_{p} s i$ ) are displayed in the bottom curves of Figure 13.

Now, the RMSE and the integral of the applied force and torque for both cases are summarized in Table 6:

Table 6: Evaluation Criteria for scenario 2

\begin{tabular}{lcccc}
\hline \hline & $R M S E_{z}(m)$ & $I N T_{z}$ & $R M S E_{\psi}($ deg $)$ & $I N T_{\psi}$ \\
Case 1 & 0.1106 & 19356 & 0.0146 & 611.19 \\
Case 2 & 0.0605 & 19831 & 0.0060 & 721.36 \\
\hline
\end{tabular}



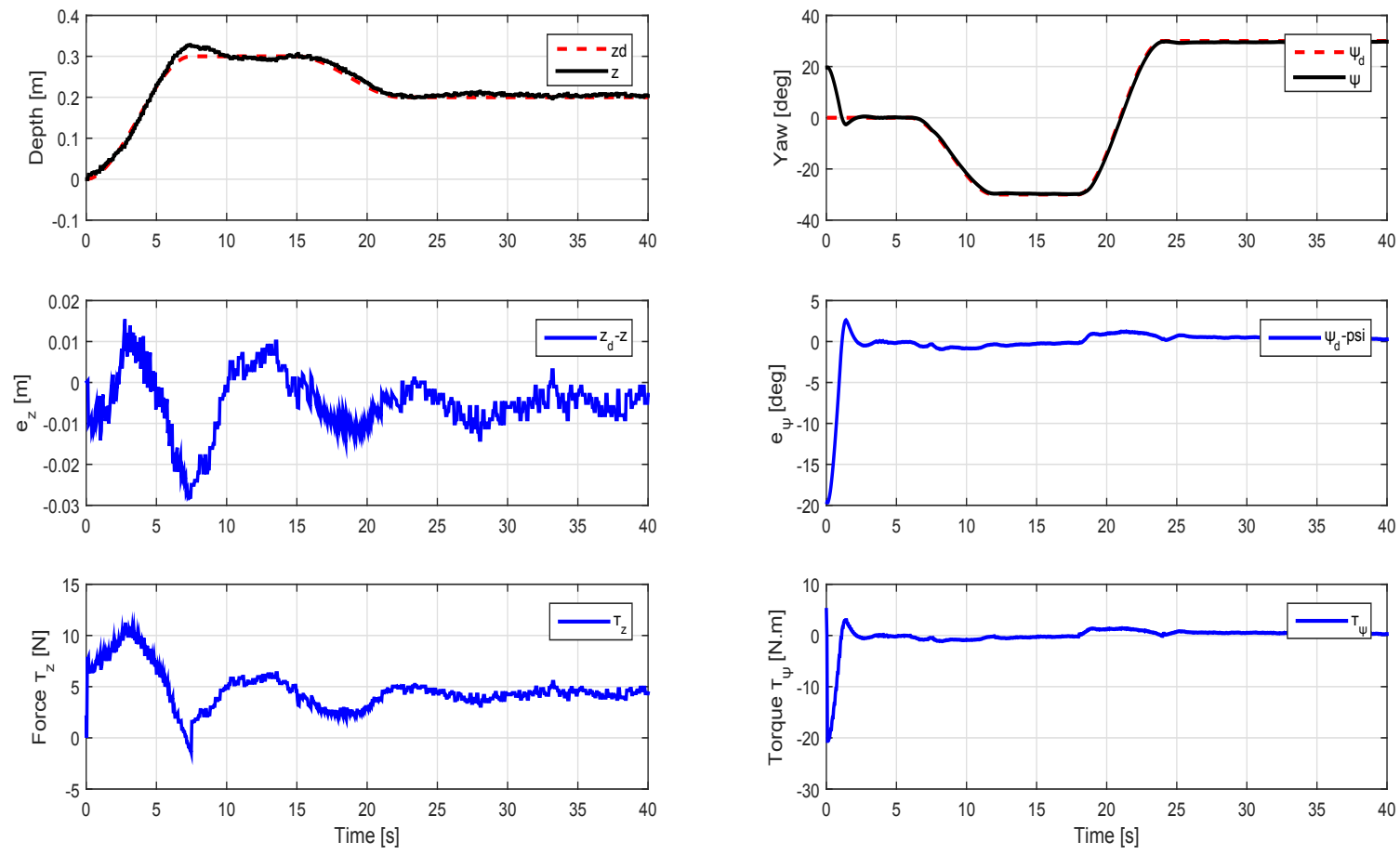

(a)

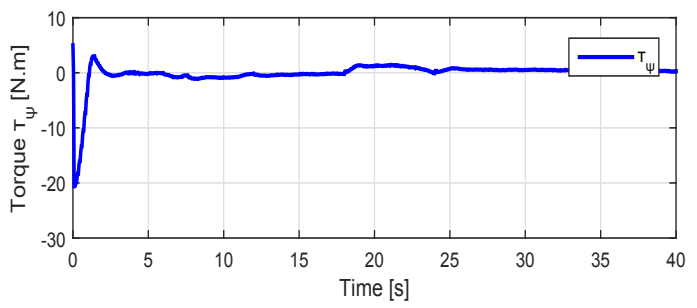

(b)

Figure 10: Experimental results of scenario 1 in the case 1: Trajectory tracking of depth and yaw versus time, Error signal of both motions and Evolution of the control inputs generated by the thrusters.
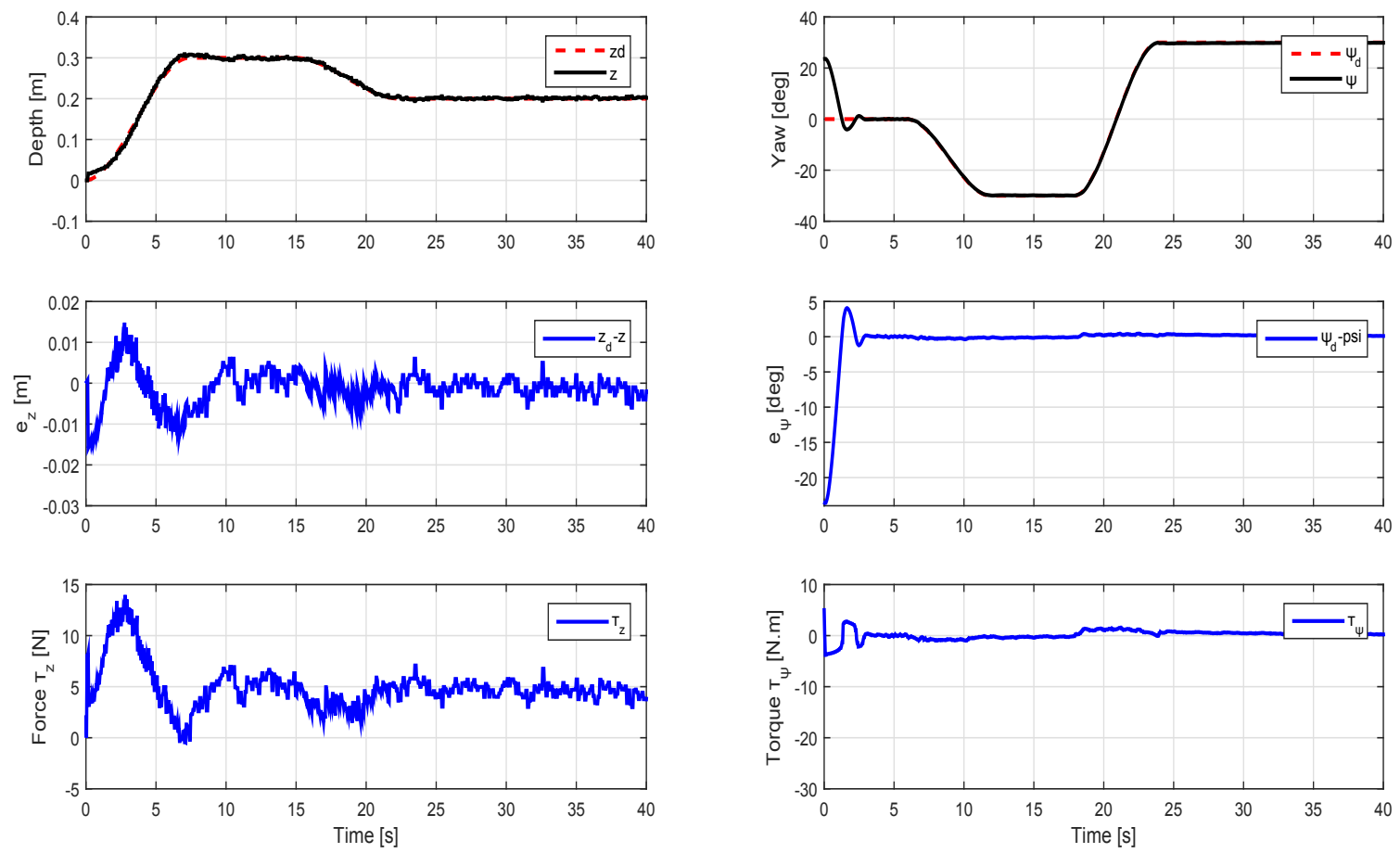

(a)

(b)

Figure 11: Experimental results of scenario 1 in the case 2: Trajectory tracking of depth and yaw versus time, Error signal of both motions and Evolution of the control inputs generated by the thrusters. 

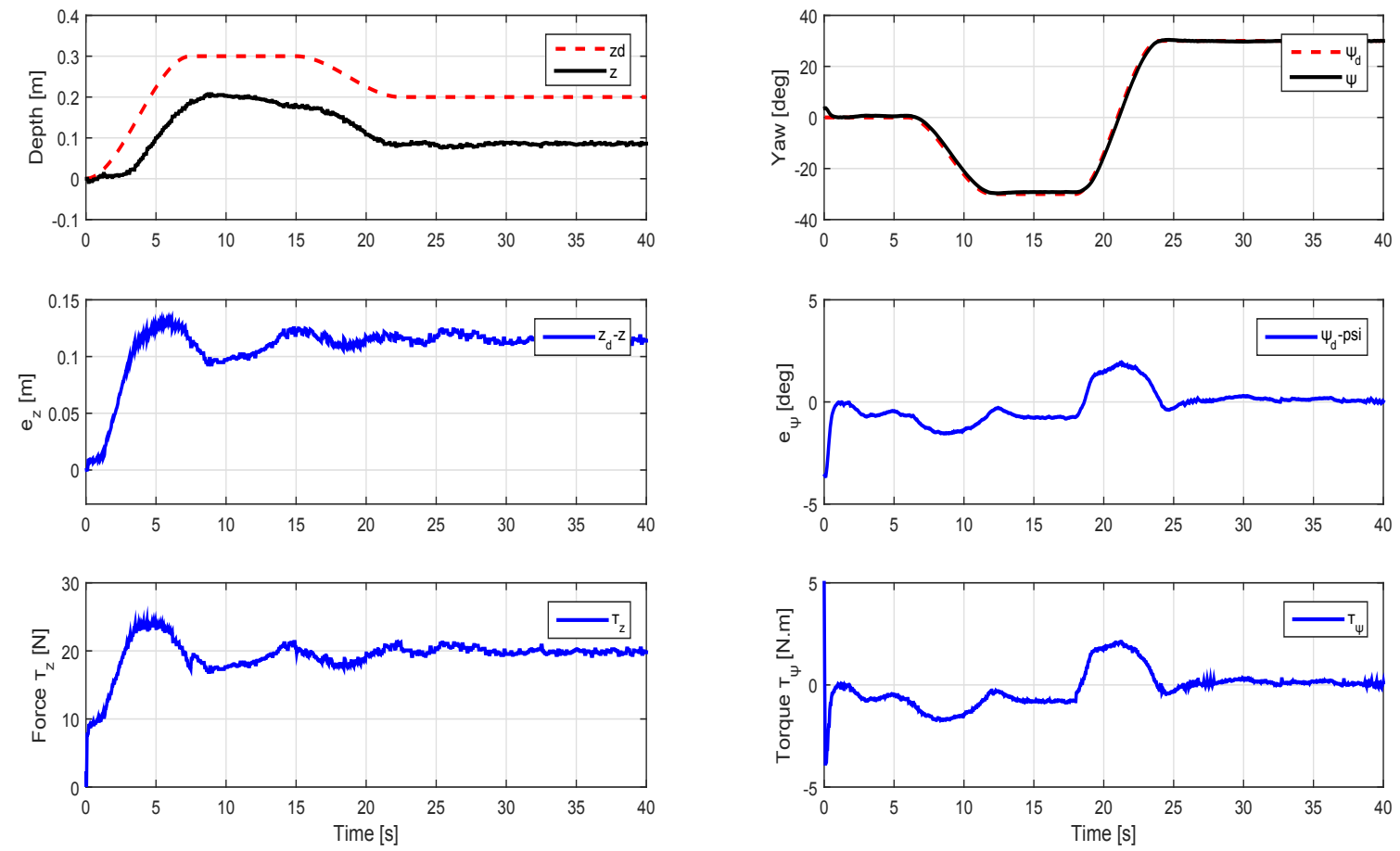

(a)

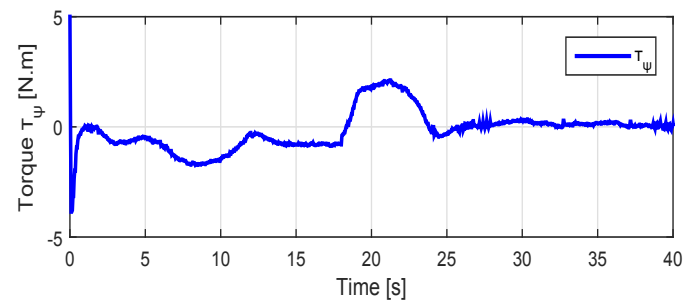

(b)

Figure 12: Experimental results of scenario 2 in the case 1: Trajectory tracking of depth and yaw versus time, Error signal of both motions and Evolution of the control inputs generated by the thrusters.
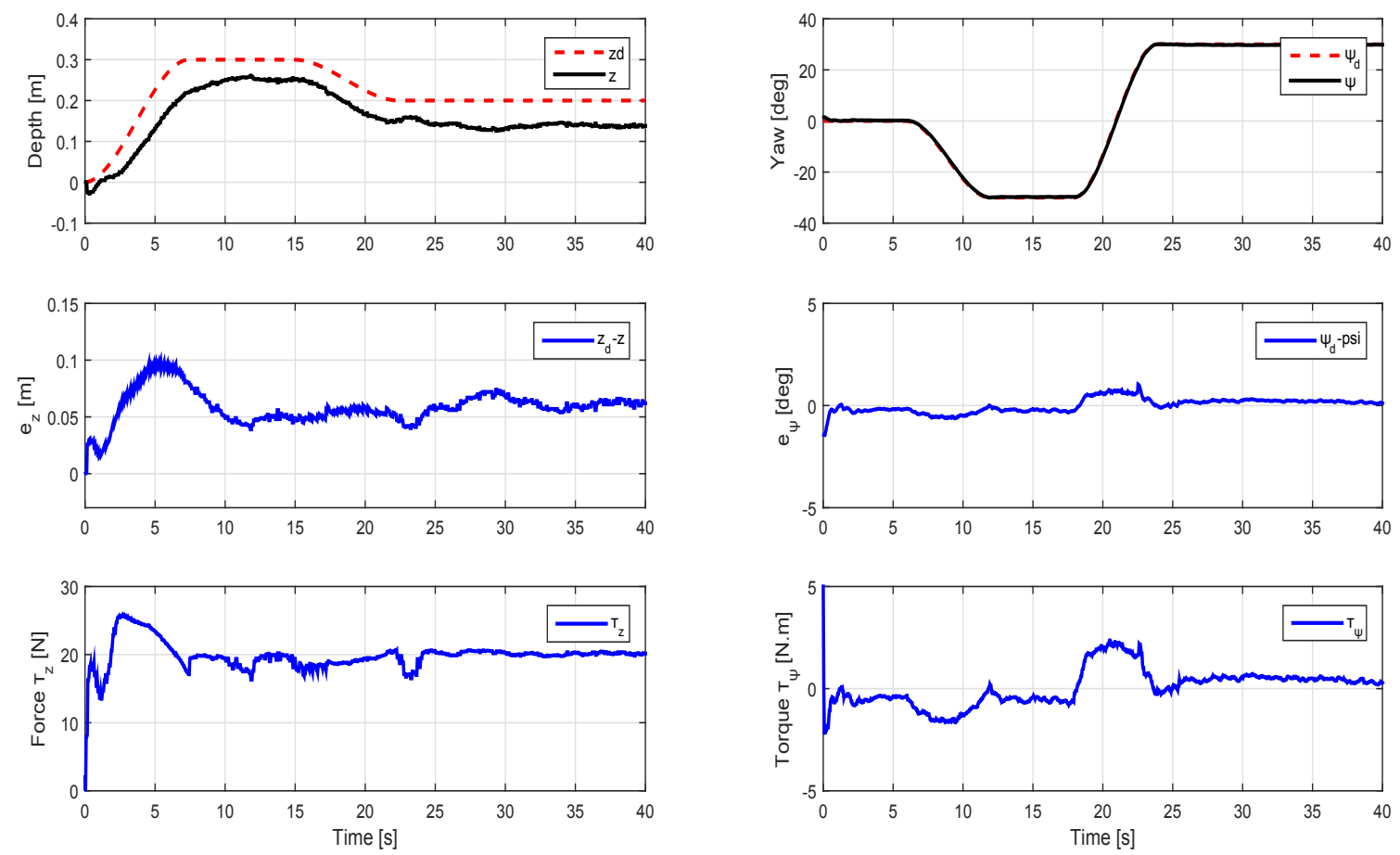

(a)

(b)

Figure 13: Experimental results of scenario 2 in the case 2: Trajectory tracking of depth and yaw versus time, Error signal of both motions and Evolution of the control inputs generated by the thrusters. 
According to Table 6 the quotients between $I N T_{z}$ and $I N T_{\psi}$ from case 1 and 2 are:

$$
\frac{19831}{19356}=1.0245 \quad \frac{721.36}{611.19}=1.1803
$$

This means that energy consumption for trajectory tracking in depth, using the controller defined in Case 2, is 1.05 times the energy consumption using the controller define in Case 1. While energy consumption for trajectory tracking in heading, using the controller defined in Case 2, is 1.27 times the energy consumption using the controller defined in Case 1. We can observe that the quotients obtained in this scenario are very similar as in the previous scenario, see equation (68). Moreover, one can notice that the closed-loop system with the nonlinear PD+ controller, represented by case 2 , is less affected. It can be observed that steady-state errors $z$ and $\psi$ are approximately $11 \mathrm{~cm}$ and $0.01 \mathrm{deg}$ for the case 1 , while for the case 2 their values are approximately $6 \mathrm{~cm}$ and $0.006 \mathrm{deg}$ respectively. Moreover, notice that the chattering is large in the second case than in the first one. This is due to the stronger compromise between performance and robustness imposed by the variable saturation case. Then, we can conclude that the proposed control strategy demonstrated a good ability to deal with parameters' uncertainties.

\section{CONCLUSION AND FUTURE WORK}

In this paper, a nonlinear PD and PD+ controllers have been proposed for depth and yaw control of underwater vehicles. The stability analysis for the resulting closed-loop system for both set-point regulation and trajectory tracking control has been addressed. The proposed controllers have been implemented for trajectory tracking in depth and yaw motions with the L2ROV underwater vehicle. The obtained experimental results demonstrate the effectiveness and the robustness of the proposed controllers towards uncertainties on the parameters of the system (damping and buoyancy changes). The future work will consist in implementing the integral term of the controller in order to improve the steady-state performance of the closed-loop system.

\section{ACKNOWLEDGEMENTS}

This work was supported by the PCP research project, in collaboration with the Tecnalia foundation. The L2ROV underwater vehicle has been funded by the Region LanguedocRoussillon council (ARPE MiniROV). The authors greatly acknowledge support of the European Union through FEDER grant $\mathrm{n}^{\circ} 49793$ for the development of the Leonard L2ROV

\section{References}

[1] Allotta B., Pugi L., Bartolini F., Ridolfi A., Costanzi R., Monni N., Gelli J., Preliminary design and fast prototyping of an Autonomous Underwater Vehicle propulsion system, Proceedings of the Institution of Mechanical Engineers Part M: Journal of Engineering for the Maritime Environment, 229 (3), pp. 248-272, 2015.
[2] Newman. Marine Hydrodynamics MIT Press. Cambridge, MA. J.N. 1977.

[3] Thor I. Fossen. Handbook of Marine Craft Hydrodynamics and Motion Control. John Wiley, 2011.

[4] H. Lamb. (1932). Hydrodynamics. Cambridge University Press. London

[5] Thor I. Fossen. Marine control systems guidance, navigation, and control of shipd, rigs and underwater vehicles. Marine Cybernetics, 2002.

[6] Carlton J., Marine Propellers and Propulsion, 2nd edition, Elsevier, 2007.

[7] E. Campos, I. Torres, O. Garcia., J. Torres and R. Lozano. Embedded system for controlling a mini underwater vehicle in autonomous hover mode CESCIT 2012, Germany, April 3-5.

[8] R. Kelly, Ricardo c. A class of nonlinear PD-type controller for robot manupulator. J Robotic Syst, 1996;13:793-802.

[9] X.Peng, Shijie Cheng, and Jinyu Wen. Application of Nonlinear PID Controller in Superconducting Magnetic Energy Storage International Journal of Control, Automation, and Systems, vol. 3, no. 2, pp. 296-301, June 2005.

[10] F. Jiang Zhiqiang Gao. An Application of Nonlinear PID Control to a Class of Truck ABS Problems Decision and Control 2001, Vol.1. Proceedings of the 40th IEEE Conference.

[11] David Bryan Marco and Anthony J. Healey Command, Control, and Navigation Experimental Results With the NPS ARIES AUV. IEEE Journal of Oceanic Engineerin, Vol. 26, No. 4, October 2001.

[12] Lionel Lapierre Robust diving control of an AUV. Ocean Engineering, 36 (2009), 92-104.

[13] Petko Kiriazov, Edwin Kreuzer, Fernando C. Pinto Robust feedback stabilization of underwater robotic vehicles. Robotics and Autonomous Systems 21 (1997) 415-423.

[14] Pivano L., Johansen T. A., Smogeli, N., A Four-Quadrant Thrust Estimation Scheme for Marine Propellers: Theory and Experiments, IEEE Trans. on Control Systems Technology, 17 (1), 2009.

[15] Ehsan Peymani, Thor I. Fossen Path following of underwater robots using Lagrange multipliers. Robotics and Autonomous Systems 2014.

[16] Petirch, J. and Stilwell D.J. Robust control for an autonomous vehicle that suppresses pitch and yaw couplings. Ocean Engineering, 38 (2011), 197-204.

[17] The Society of Naval Architects and Marine Engineers. Nomenclature for Treating the Motion of a Submerged Body Through a Fluid. IN: Technical and Research Bulletin No. 1-5.

[18] Thor I. Fossen. Guidance and Control of Ocean Vehicles. John Wiley and Sons, Norway, Secondedition, 1999.

[19] H. Goldstein, C.P. Poole and J.L Safko. Classical Mechanics. Addison Wesley Series in Physics,Adison-Wesley, USA, second edition, 1983.

[20] J.E. Marsden. Elementary Classical Analysis. W.H. Freeman and Company, San Francisco, 1974.

[21] A Visioli. Optimal tuning of pid controllers for integral and unstable processes. Proceedings of the IEEE, Part D, 148(2):180-194, 2001. 